\title{
Functionalized graphene in quantizing magnetic field: The case of bunched impurities
}

\author{
P. G. Silvestrov \\ Institute for Mathematical Physics, TU Braunschweig, 38106 Braunschweig, Germany \\ and Physics Department and Dahlem Center for Complex Quantum Systems, Freie Universität Berlin, 14195 Berlin, Germany
}

(Received 12 August 2014; revised manuscript received 3 November 2014; published 17 December 2014)

\begin{abstract}
Resonant scattering at the atomic absorbates in graphene was investigated recently in relation with the transport and gap opening problems. Attaching an impurity atom to graphene is believed to lead to the creation of unusual zero-energy localized electron states. This paper aims to describe the behavior of the localized impurity-induced levels in graphene in a quantizing magnetic field. It is shown that in the magnetic field the impurity level effectively hybridizes with one of the $n=0$ Landau level states and splits into two opposite-energy states. The new hybridized state is doubly occupied, forming a spin singlet and reducing the polarization of a quantum Hall ferromagnet in undoped graphene. Taking into account the electron-electron interaction changes radically the spectrum of the electrons surrounding the impurity, which should be seen experimentally. While existing publications investigate graphene uniformly covered by adatoms, here we address a possibly even more experimentally relevant case of the clusterized impurity distribution. The limit of a dense bunch of the impurity atoms is considered, and it is shown how such a bunch changes the spectrum and spin polarization of a large dense electron droplet surrounding it. The droplet is encircled by an edge state carrying a persistent current.
\end{abstract}

DOI: 10.1103/PhysRevB.90.235130

PACS number(s): 73.22.Pr, 73.43.-f, 81.05.ue

\section{INTRODUCTION}

First theoretical works following the discovery of graphene almost a decade ago [1,2] were concentrated on the relativistic character of its electronic spectrum. It was, however, quickly realized that this two-dimensional material can offer a plethora of interesting effects, going far beyond the quasirelativistic behavior of the bulk electrons [3]. One class of such effects comes from the investigation of the graphene edges, where for example in the case of zigzag edge one finds a band of dispersionless zero-energy edge states. Remarkably, as was shown already in the early work [4], even the shortest possible edge in graphene, which is the closed edge of the hole created by removing a single carbon atom, is sufficient to create a single localized zero-energy electron state with the algebraic wave function $\psi \sim 1 /(x+i y)$. The existence of such localized low-energy states with a power-law coordinate dependence of the density is an indication of the resonant scattering at the Dirac point in graphene.

A number of theoretical papers have addressed the properties of graphene with resonant impurities [5-19]. Experimentally, a way to create the strong atom-size small impurities is by chemical functionalization of graphene by impurity adatoms (see, e.g., Refs. [20-23]). Mobility inherent for adatoms allowed to put forward theoretical proposals, suggesting the impurities sublattice ordering caused by their Casimir-type interaction [5-10]. This ordering would lead to the controllable opening of the gap in the electron's spectrum, highly desired for the graphene electronics. Resonance at the Dirac point in this case is necessary in order to make the Casimir interaction sufficiently long ranged.

The choice of the theoretical model describing the impurity requires a special attention. Adding a large potential at a certain carbon atom, a scheme assumed by most of the authors, does not work [18] since this requires the use of unrealistically large impurity potential (hundreds of eV!). A more realistic model, which is adopted in this paper, was suggested in Refs. [12,13], where the adatom is treated not as a potential scatterer, but as a quantum level tunnel coupled to one of the carbon sites. The tunnelling amplitude between the adatom and carbon atom is of order $\sim 1 \mathrm{eV}$, but the energy of the electron's level at the impurity turns out to be very close to the Dirac point, thus leading to the resonance scattering in undoped graphene. Density functional theory analysis [12-14] indicates that this situation may indeed be realized for several kinds of impurity atoms.

In this paper, we consider graphene functionalized by the impurity atoms in a strong perpendicular magnetic field with the special emphasis on the investigation of interaction between electrons surrounding the impurities, a combination of problems never touched in the existing literature. Several interesting and potentially experimentally relevant results for both the electron spectrum and the spin density caused by the joint effect of the impurity and magnetic field will be presented.

A peculiar feature of graphene in a magnetic field is the existence of zero-energy Landau levels with fully isospin (sublattice) polarized electron states [24]. Thus, in the presence of impurity one needs to analyze the coexistence of two kinds of zero-energy states, localized due to the impurity and due to the quantum cyclotron motion.

We use the model of Ref. [12] to describe the impurity atoms and assume that the energy of the impurity level is small compared to the Landau level splitting, which means a particlehole-symmetric limit. Both single- and many-impurities problems, with and without electron-electron interaction, will be considered.

The paper starts with the investigation of the zero-energy state induced by a single impurity in the magnetic field in case of noninteracting electrons. Remarkably in this case we found a simple analytical solution for the wave function and energy. The solution is approximate, with the small parameter being the inverted large logarithm (of the Larmor radius divided by the graphene lattice spacing), but it is sufficient to describe the wave functions and the occupation of the eigenstates. The important properties of the result are as follows: First, the new resonant states are indeed 
impurity-induced states in the graphene layer and not the states localized on the impurity. The probability to find the electron in this state in graphene plane increases in the vicinity of the impurity, but the probability to stay exactly on the impurity atom is parametrically small. Second, both the $n=0$ Landau level states and the impurity states without magnetic field have exactly zero energies. That was the reason to expect both kinds of states in undoped graphene to be half occupied and spin polarized [25-27]. Now, when both the impurity and the magnetic field are present, the impurity-induced states acquire a finite energy and their number is doubled since the non-zero-energy states may only appear in pairs in the particle-hole-symmetric limit. Doubling the number of levels becomes possible because the impurity level in the magnetic field gets hybridized with the $n=0$ Landau level electron state most coupled to the impurity. The negative-energy impurity-induced level is doubly occupied, thus reducing the total polarization of the quantum Hall ferromagnetic state of graphene in a magnetic field.

A realistic description of the spin-polarization effects in quantum Hall regime is impossible without taking into account interaction between electrons. That is why, after solving the noninteracting problem, we proceed with the calculation of the spectrum, taking into account the exchange electronic interaction in the Hartree-Fock approximation. As expected, adding the exchange interaction increases the Zeeman splitting of the $n=0$ Landau level in graphene by 1 to 2 orders of magnitude. What is new and interesting, we found that for the states surrounding the impurity the splitting of upand down-spin states depends on the angular momentum number $m$. The electron with $m=1$, which is not directly connected to the impurity, gets the smallest interactioninduced Zeeman splitting and thus the smallest excitation energy.

In this paper, we treat the electron-electron interaction in the Hartree-Fock approximation in the Hilbert space restricted to the $n=0$ Landau level. Strictly speaking, this approach accounts fully for the electron's interaction only in the first order. This is justified because the electron-electron interaction in graphene effectively is not very strong (and even may be further suppressed by covering the sample by the material with large dielectric constant [28-31]). Moreover, all the interaction-related predictions of this paper are of qualitative nature and should remain intact after taking into account higher-order corrections in case of moderately strong interaction.

In addition to the single impurity, we consider the group of several (many) impurity atoms attached to the graphene sheet in a strong magnetic field. Existing publications investigate multiple impurities distributed with a uniform density over the graphene sample, leaving aside the potentially experimentally relevant case of bunched impurities. To fill this gap, we consider several adatoms forming a dense bunch, such that the distance between any two adatoms is small compared to the Larmor radius (but still large compared to the lattice spacing). The Casimir interaction between impurities [5] favors the configurations with the impurities coupled to the same sublattice of graphene (Kekule ordering). Consequently, we investigate the bunch of impurities coupled all to the same sublattice of graphene. The main result in this case is that a dense bunch of many impurities/adatoms changes the electronic structure within a large area of a graphene flake around it. As was written above, an impurity in the magnetic field creates a couple of localized states by hybridizing the singular $\sim 1 /(x+i y)$ state of Ref. [4] with the $n=0$ Landau level state mostly connected to the impurity. In the case of many impurities, each of them tends to create such a couple of hybridized levels, for which it needs the $n=0$ Landau level state. Thus, many $n=0$ states with the angular momentum $m \geqslant 0$ become hybridized, leading to the formation of a spin-unpolarized circular droplet of electrons residing on one of the graphene sublattices around the bunch of impurities. The energies of electrons, forming the droplet, although nonzero, unlike the energies of other $n=0$ electrons, decrease fast with the increasing angular momentum. Namely, increasing $m$ by one leads to a decrease of the energy by a small factor $\sim\left\langle r_{a b}\right\rangle / l$, where $\left\langle r_{a b}\right\rangle$ is the typical distance between impurity atoms in the bunch and $l$ is the Larmor radius.

Taking into account the exchange electron-electron interaction changes the properties of the unpolarized electron droplet surrounding the bunch of impurities in two important ways. First, the energies of electrons forming the droplet now become all of the same order of magnitude $\sim e^{2} / l$. (The energies of electrons with larger angular momentum $m$ are still smaller. But, the smallness is due to a pure numerical factor in the energy $\varepsilon_{m}$.) The second feature is that after the exchange interaction is taken into account, electrons surrounding the droplet start to feel the existence of electrons inside the droplet. More precisely, as we will show, the electrons from the $n=0$ Landau level staying outside the droplet interact via the exchange interaction with each other, but not with the electrons from the droplet. Thus, the circumference of the droplet serves as an edge for the outside electrons, leading to a circular edge current.

Resonant impurities in graphene in a magnetic field were considered recently in Ref. [19]. However, this paper is concentrated on transport properties and does not investigate the electronic spectrum and spin structure. What is more important, authors of Ref. [19] do not consider electrons' interaction in graphene.

The paper is organized as follows. In Sec. II, we introduce the Hamiltonian for graphene and the attached impurity atom and solve the single-impurity problem in the magnetic field for noninteracting electrons. To do this, we first present the solution for Landau level states in graphene in the polar gauge. The important supplementary information for Sec. II is included in Appendix A. In Sec. III, we consider the singleimpurity problem with the electron-electron interaction taken into account. The main part of the section is devoted to the explanation of the structure of exchange interaction in the case of the particle-hole-symmetric Dirac equation in graphene and to the discussion of how differently the states with zero-energy and non-zero-energy experience the exchange interaction in this case. Details of calculations for this section are given explicitly in Appendix B. In Sec. IV, we consider the problem of many close (closer than the Larmor radius) impurity atoms. Section IV A deals with the exchange Coulomb interaction in case of a bunch of impurities. Calculational details for Sec. IV are given in the Appendices C and D. Section V presents the conclusions. 


\section{SINGLE IMPURITY}

A graphene plane with an impurity atom chemically bonded to it is described by the Hamiltonian

$$
H=t \sum_{\langle i, j\rangle}\left(a_{i}^{\dagger} b_{j}+b_{j}^{\dagger} a_{i}\right)+U\left(a_{0}^{\dagger} d+d^{\dagger} a_{0}\right)+\varepsilon_{d} d^{\dagger} d,
$$

where operators $a_{j}^{\dagger}, b_{j}^{\dagger}$ create an electron on the $j$ th site of the triangular sublattices $A$ and $B$ of the honeycomb lattice of graphene, and $d^{\dagger}$ creates an electron at the impurity atom attached to the carbon atom of the sublattice $A$ with $j=0$. Electrons hop from a site of one sublattice to the nearest sites of another sublattice with the matrix element $t \approx 2.7 \mathrm{eV}$. Hopping matrix element between the impurity and the carbon atom nearest to it has the same order of magnitude $U \sim t$. Crucial for us is the prediction of Refs. [12,13] that the energy of the electron at the impurity may be anomalously small, $\varepsilon_{d} \ll$ $t, U$, for several popular choices of the impurity atom. Only in the case of the impurity level being very close to the Dirac point, the model (1) leads to resonant scattering of low-energy electrons, which is a necessary ingredient for Refs. [5-19]. That is why in this paper we will always assume a negligibly small impurity level energy $\varepsilon_{d}=0$.

Single-particle eigenstates of the Hamiltonian (1) are created by the operator

$$
\hat{\Phi}^{\dagger}=f_{d} d^{\dagger}+\sum_{n} f_{n} \hat{\Psi}_{n}^{\dagger}, \quad \hat{\Psi}_{n}^{\dagger}=\sum_{j}\left(u_{n j} a_{j}^{\dagger}+v_{n j} b_{j}^{\dagger}\right) .
$$

Here, $f_{d}$ is a probability amplitude to find the electron on the impurity atom and $f_{n}$ are the probability amplitudes to find the electron in the $n$th eigenstate of the pure graphene Hamiltonian. These eigenstates are determined by two complex amplitudes $u_{n j}$ and $v_{n j}$ describing the electron residing on one of the graphene sublattices $A$ or $B$ in the unit cell $j$. As usual, the same unit-cell amplitudes $u_{n j}$ and $v_{n j}$ are combined into a (pseudo)spinor $\Psi_{n}\left(\mathbf{r}_{j}\right)$. The low-energy behavior of the wave function $\Psi_{n}$ is captured by two spinor envelope functions $\psi_{n}$ and $\psi_{n}^{\prime}$ :

$$
\Psi_{n}\left(\mathbf{r}_{j}\right)=\left(\begin{array}{c}
u_{n j} \\
v_{n j}
\end{array}\right)=e^{i \mathbf{K r}_{j}} \psi_{n}\left(\mathbf{r}_{j}\right)+e^{i \mathbf{K}^{\prime} \mathbf{r}_{j}} \psi_{n}^{\prime}\left(\mathbf{r}_{j}\right),
$$

where vectors $\mathbf{K}$ and $\mathbf{K}^{\prime}=-\mathbf{K}$ are directed to two inequivalent corners of the Brillouin zone.

In order to proceed with solving the impurity problem in Eqs. (1) and (2), one first needs to find the eigenfunctions of the clean graphene Hamiltonian in a magnetic field. The latter may be added into the Hamiltonian (1) by introducing the coordinate-dependent hopping matrix elements $t_{i j}$ with properly chosen phases. We, however, will be interested only in the low-energy limit, when the two envelope functions $\psi, \psi^{\prime}$ become the eigenfunctions of two decoupled massless Dirac Hamiltonians [3] with the magnetic field entering via the covariant derivative $\mathbf{p} \rightarrow \mathbf{p}-(e / c) \mathbf{A}$. In the polar gauge, $\mathbf{A}=(B y / 2,-B x / 2,0)$ the two Dirac Hamiltonians for $K$ and $K^{\prime}$ valleys become

$$
\mathcal{H}=i \varepsilon_{B}\left(\tau_{-} Q-\tau_{+} Q^{+}\right), \quad \mathcal{H}^{\prime}=i \varepsilon_{B}\left(\tau_{-} Q^{+}-\tau_{+} Q\right) .
$$

Here, $\tau_{ \pm}=\left(\tau_{x} \pm i \tau_{y}\right) / 2$ and $\tau_{x, y}$ are the Pauli matrices in the pseudospin space, and $\varepsilon_{B}=\hbar v_{F} / l$, where $l=\sqrt{\hbar c / e B}$ is the
Larmor radius. The Fermi velocity in graphene is determined by the hopping matrix element $t$ in Eq. (1) and the carboncarbon distance $d, \hbar v_{F}=3 d t / 2$. The creation and annihilation operators $Q^{+}$and $Q$, in terms of the dimensionless complex coordinate $z=(x+i y) / l$, have the form

$$
Q^{+}=\frac{1}{\sqrt{2}}\left(-2 \frac{\partial}{\partial z^{*}}+\frac{z}{2}\right), \quad Q=\frac{1}{\sqrt{2}}\left(2 \frac{\partial}{\partial z}+\frac{z^{*}}{2}\right) .
$$

They satisfy the "oscillator" commutation relations $\left[Q, Q^{+}\right]=$ 1. To find the eigenfunctions of $\mathcal{H}$ and $\mathcal{H}^{\prime}$ we introduce a set of (normalized) functions, $n, m>0$,

$$
\phi_{n, m-n}=\frac{\left(-Q^{+}\right)^{n} z^{* m} e^{-z z^{*} / 4}}{\sqrt{2 \pi n ! 2^{m} m !}} .
$$

Here, the second index is responsible for the angular behavior $\phi_{n, k} \sim\left(z^{*} /|z|\right)^{k}$ and may be both positive and negative. The two sets of eigenfunctions are now for $n>0$ (the $n=0$ case should be considered separately)

$$
\psi_{ \pm n, k}=\left(\begin{array}{c}
\frac{1}{\sqrt{2}} \phi_{n, k} \\
\frac{\mp i}{\sqrt{2}} \phi_{n-1, k+1}
\end{array}\right), \quad \psi_{ \pm n, k}^{\prime}=\left(\begin{array}{c}
\frac{1}{\sqrt{2}} \phi_{n-1, k+1} \\
\frac{\mp i}{\sqrt{2}} \phi_{n, k}
\end{array}\right) .
$$

The corresponding energies $\varepsilon_{ \pm n}$ and $\varepsilon_{ \pm n}^{\prime}$ coincide and depend only on the Landau level number $n$ :

$$
\varepsilon_{ \pm n}=\varepsilon_{ \pm n}^{\prime}= \pm \sqrt{n} \varepsilon_{B} .
$$

For each value of $n$, only two of the solutions (7), $\psi_{ \pm n, 0}(z=$ $0)=\psi_{ \pm n,-1}^{\prime}(z=0)=(1 / \sqrt{2 \pi}, 0)$, have nonzero upper component at the origin and can be coupled to the impurity attached to the carbon atom $A$ with $\mathbf{r}_{j}=0$.

The most interesting for us will be the zero-energy states from the $n=0$ Landau level. In undoped graphene, one spin component of this level is fully occupied by electrons and the other spin component remains empty, leading to a strongly spin-polarized state often called a quantum Hall ferromagnet. Wave functions for the $n=0$ Landau level have also a very special form, with electrons from the $\mathbf{K}$ valley residing solely on the sublattice $A$ and electrons from the $\mathbf{K}^{\prime}$ valley on the sublattice $B$. Corresponding envelope functions are

$$
\psi_{0, m}=\left(\begin{array}{c}
\phi_{0, m} \\
0
\end{array}\right), \quad \psi_{0, m}^{\prime}=\left(\begin{array}{c}
0 \\
\phi_{0, m}
\end{array}\right)
$$

Only one state from the $n=0$ level, $\psi_{0,0}$, has a nonvanishing upper component at $\mathbf{r}=0$ and can be coupled to the impurity. All the other zeroth Landau level states are decoupled from the carbon site connected to the impurity and thus remain the exact zero-energy eigenstates of the full Hamiltonian (1).

Similarly, for each of the other Landau levels, $n \neq 0$, one may choose a basis with only one state having nonvanishing probability amplitude at the impurity. This single coupled state has to be a superposition of solutions belonging to different valleys [Eq. (4)], each being nonzero at the carbon atom coupled to the impurity. Explicitly, the subset of eigenmodes of the graphene hopping Hamiltonian in the magnetic field coupled to the impurity is, for $n \neq 0$,

$$
\Psi_{n}=\frac{e^{i \mathbf{K r}}}{2}\left(\begin{array}{c}
\phi_{|n|, 0} \\
\frac{n}{i|n|} \phi_{|n|-1,1}
\end{array}\right)+\frac{e^{i \mathbf{K}^{\prime} \mathbf{r}}}{2}\left(\begin{array}{c}
\phi_{|n|-1,0} \\
\frac{n}{i|n|} \phi_{|n|,-1}
\end{array}\right) .
$$


This set is completed by adding the $n=0$ solution $\Psi_{0}=$ $e^{i \mathbf{K r}} \psi_{0,0}$ [Eq. (9)]. [Another combination of two spinor functions from Eq. (10) with opposite relative sign vanishes at the carbon site coupled to the impurity atom.]

All wave functions $\Psi_{n}$ [Eq. (10)] are equally coupled to the impurity $\Psi_{n}(0)=(1 / \sqrt{2 \pi}, 0)$. Thus, the problem of describing the effect of resonant impurity in graphene reduces to solving the problem with a single-impurity level equally coupled to a discrete ladder of special graphene states in magnetic field. The sum over $n$ in Eq. (2) now includes only the ladder states $\Psi_{n}$. The energy and the complex amplitudes $f_{n}, f_{d}$ satisfy the equations

$$
\left(\varepsilon-\varepsilon_{n}\right) f_{n}=V_{d} f_{d}, \quad\left(\varepsilon-\varepsilon_{d}\right) f_{d}=V_{d} \sum_{n} f_{n} .
$$

Here, $V_{d}=3^{\frac{3}{4}} U d /\left(2 \pi^{\frac{1}{2}} l\right)$. When deriving $V_{d}$ one should remember that the Hamiltonian (1) acts in the lattice space, while the ladder states (10) are the normalized functions of the continuous coordinates. Equations (11) may be rewritten as an algebraic equation for the energy and expression for all amplitudes $f_{n}$ through a single-"impurity" amplitude $f_{d}$ :

$$
\varepsilon-\varepsilon_{d}=\sum_{n} \frac{V_{d}^{2}}{\varepsilon-\varepsilon_{n}}, \quad f_{n}=\frac{V_{d}}{\varepsilon-\varepsilon_{n}} f_{d} .
$$

The probability to find the electron on the impurity is found from the normalization condition, yielding

$$
f_{d}^{2}=\frac{1}{1+V_{d}^{2} \sum 1 /\left(\varepsilon-\varepsilon_{n}\right)^{2}} .
$$

A graphical solution of the energy equation (12) is shown in Fig. 1. Energy levels are the energies at which the straight solid line representing the left-hand side of the equation crosses the multiple solid lines showing the sum of hyperbolae $1 /\left(\varepsilon-\varepsilon_{n}\right)$ in the right-hand side. The impurity-induced states correspond to the two crossings most close to zero energy.

Details of the explicit analytical solution of Eqs. (12) are given in the Appendix A. Here, we show only the results for the in-plane wave function $\Psi_{S}=\sum f_{n} \Psi_{n}$, and energy of the singular impurity-induced state

$$
\Psi_{S_{ \pm}}=\left(\begin{array}{c}
e^{i \mathbf{K r} r \frac{e^{-z z^{*} / 4}}{2 \sqrt{\pi}}} \\
\pm i \operatorname{Im}\left[\frac{e^{i \mathbf{K r}}}{z}\right] \frac{e^{-z z^{*} / 4}}{\sqrt{2 \pi L}}
\end{array}\right), \quad \varepsilon_{S_{ \pm}}=\frac{ \pm \varepsilon_{B}}{2 \sqrt{L}} .
$$

The analytical solution is found in the large logarithm limit $L=\ln (l / d) \gg 1$, i.e., in case of Larmor radius $l$ very much exceeding the carbon-carbon distance $d$. However, the resulting approximate wave function allows us to extract many qualitative features of the exact solution. As it should be for the eigenfunctions of the particle-hole-symmetric Hamiltonian (1) for vanishing $\varepsilon_{d}$, there are two solutions $\Psi_{S_{ \pm}}$with opposite signs of energy. Electron in the state described by Eq. (14) can hop to the impurity attached at $\mathbf{r}=0$ to the carbon atom from the sublattice $A$. However, the probability to find an electron on the impurity, found via Eq. (13), is small $f_{d}^{2}=\sqrt{3} \pi t^{2} /\left(4 U^{2} L\right) \ll 1$, and most of the time the electron spends in the graphene plane (remember that $U \sim t$ ). The electronic density corresponding to Eq. (14) is shown in Fig. 2.

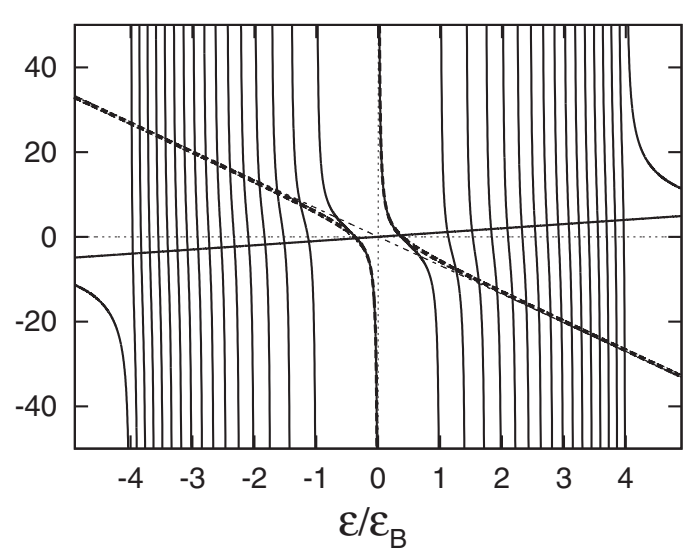

FIG. 1. Finding graphically the energy from the first equation (12). (Energy in units of $\varepsilon_{B}$ and $V_{d}=1$.) Straight solid line with small positive slope shows the left-hand side of the equation. Other solid lines show the right-hand side of Eq. (12), having a pole at $\varepsilon=0$ and other poles at each $\varepsilon= \pm \sqrt{n} \varepsilon_{B}$. Each crossing of two solid lines corresponds to an energy level. Impurity-induced localized states $\Psi_{S_{ \pm}}$correspond to two crossings closest to the zero energy. In addition, there are two crossings far to the right and far to the left from the energy segment shown in the figure (well outside the energy band of graphene). These crossings correspond to the bonding and antibonding states of the impurity atom and a single carbon atom closest to it. They are responsible for the creation of the true chemical bond between the two atoms. Thick dashed line with a single pole at $\varepsilon=0$ shows the approximate form of the energy equation (12) right-hand side described in Appendix A.

The wave functions $\Psi_{S_{ \pm}}$[Eq. (14)] have a clear physical interpretation. The two states $\Psi_{S_{ \pm}}$may be thought of as equal weight superpositions of two simple states, which contribute

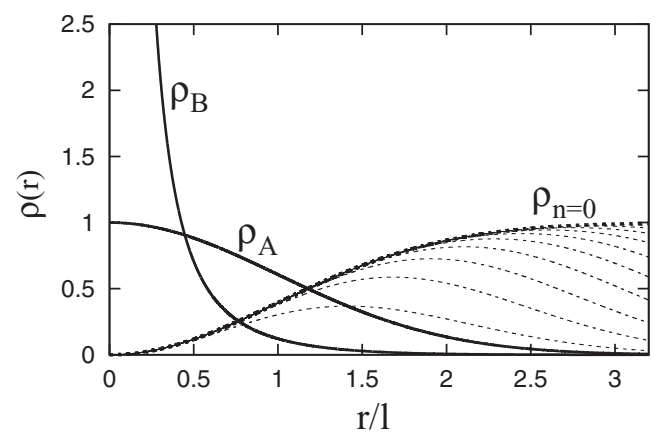

FIG. 2. Radial dependence of the electron density at two sublattices $\rho_{A}$ and $\rho_{B}$ for the impurity-induced state $\Psi_{S_{-}}$[Eq. (14)]. The density is doubled due to two spin components and normalized such that a single occupied Landau level has $\rho=1$. For drawing we choose $L=\ln (l / d)=5$. The thick dashed line shows the density $\rho_{n=0}$ of the $n=0$ Landau level from sublattice $A$, valley $K$, with one spin orientation and having angular momenta $m=1,2,3, \ldots$ (the states not affected by the impurity). Thin dashed lines show how this density is built by adding one by one electrons with $m=1, m=2$, etc. Together $\rho_{A}$ and $\rho_{n=0}$ give a constant charge density of a fully occupied Landau level. However, these constant density electrons have a very nontrivial exchange interaction, discussed in Sec. III. The singular increase of density in state $\Psi_{S}$ at sublattice $B, \rho_{B} \sim 1 / r^{2}$, is fully compensated by the decrease of density in Landau levels with $n>0$, as follows from the particle-hole symmetry. 
to their upper and lower components, respectively. First is the $n=0$ Landau state $\psi_{0,0}$ [Eq. (9)], having only one nonvanishing component (upper). The lower component of Eq. (14) comes from the zero-energy localized state found in Ref. [4] with the large-distance cutoff at the Larmor radius $r \sim l$. Note that the lower component of $\Psi_{S_{ \pm}}$contains similar contributions from both the $K$ and $K^{\prime}$ valleys.

The energies of the states $\Psi_{S_{ \pm}}$in Eq. (14) are also small compared to the Landau level splitting $\varepsilon_{B}$, but only as an inverse square root of the large logarithm. A chemical potential in undoped graphene coincides with the Dirac point. This suggests that both spin components of the level $\varepsilon_{S_{-}}$ are occupied and form a spin singlet, thus preserving the particle-hole symmetry and electrical neutrality of the system, graphene plus impurity. Taking into account spin of the electron leads also to a small Zeeman splitting of all energy levels introduced in this section. Without the impurity, the simplest choice of occupation of Landau levels in graphene is to fully occupy both $K$ and $K^{\prime}$ valley components of the $n=0$ level with spin down while keeping empty their spin-up counterpart. This quantum Hall ferromagnetic state minimizes the Zeeman energy of half-occupied $n=0$ Landau level in neutral undoped graphene. Thus, according to Eq. (14) each adatom tends to lower the polarization of the quantum Hall ferromagnet by one electron spin, i.e., by $\frac{1}{2}$. As will be shown in the following section, this scheme of occupying the $n=0$ Landau level remains intact after taking into account electron-electron interactions.

\section{SINGLE IMPURITY WITH INTERACTION}

A bare Zeeman splitting for electrons in graphene is linear in magnetic field $E_{Z}=g \mu_{B} B$. The splitting of Landau levels $\varepsilon_{B} \propto \sqrt{B}$ scales as the square root of the magnetic field and formally grows much slower with the increasing field than $E_{z}$. However, for any realistic values of the magnetic field, the Zeeman splitting is negligibly small compared to the interlevel distance. For example, for the magnetic field $10 \mathrm{~T}$, $\varepsilon_{B} / E_{Z} \approx 70$ [32], while the typical interaction energy $e^{2} / l \sim$ $\left(e^{2} / \hbar v_{F}\right) \varepsilon_{B} \sim \varepsilon_{B}$. Thus, similarly to what happens in the conventional semiconductor heterostructures, the spin physics in magnetic field in graphene is dominated by the electronic interaction. The strong renormalization of the Zeeman splitting is seen already in the Hartree-Fock approximation and is due to the exchange interaction. Direct interaction, i.e. Hartree, is trivial even in the presence of the impurity atom since we are working in the particle-hole-symmetric limit (see Fig. 2 and discussion in the caption).

We begin this section with showing that due to the particlehole symmetry of the Dirac equation, the exchange interaction acts very differently on the electrons from the $n=0$ and $n \neq 0$ Landau levels in graphene. To calculate the exchange energy of the electron in a state $\Psi$,

$$
\varepsilon_{\text {exch }}=-\int \Psi^{\dagger}(\mathbf{r}) \frac{e^{2}}{\left|\mathbf{r}-\mathbf{r}^{\prime}\right|} \rho\left(\mathbf{r}, \mathbf{r}^{\prime}\right) \Psi\left(\mathbf{r}^{\prime}\right) d \mathbf{r} d \mathbf{r}^{\prime},
$$

one needs to find the projection operator onto the occupied states

$$
\rho\left(\mathbf{r}, \mathbf{r}^{\prime}\right)=\sum_{n} v_{n} \Psi_{n}(\mathbf{r}) \Psi_{n}^{\dagger}\left(\mathbf{r}^{\prime}\right)
$$

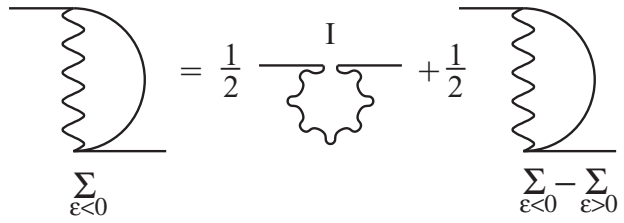

FIG. 3. Diagrammatic presentation of the exchange energy. Wavy line shows the Coulomb interaction $e^{2} /\left|\mathbf{r}-\mathbf{r}^{\prime}\right|$. Summation over the complete set of states in the first term in the right-hand side gives unity, or $\delta$ function.

where $v_{n}$ is the occupation number for the state $n$, taking values 0 or 1 (we consider only the zero-temperature limit in this paper). In the case of undoped graphene with $\varepsilon_{F}=0$, it is convenient to split the summation in Eq. (16) into a sum over the complete set of all states and a difference of two sums over occupied and empty states, leading to

$$
\rho\left(\mathbf{r}, \mathbf{r}^{\prime}\right)=\frac{\mathbf{I}}{2}+\sum_{i} \frac{2 v_{i}-1}{2} \Psi_{i}(\mathbf{r}) \Psi_{i}^{\dagger}\left(\mathbf{r}^{\prime}\right) .
$$

The unity operator $\mathbf{I} \sim \delta\left(r-r^{\prime}\right)$ here causes the uniform shift of all onsite energies in the Hamiltonian (1) (see Fig. 3) and may be ignored.

Since the signs of two sublattice components of the graphene Hamiltonian eigenfunctions may always be chosen as $u_{\varepsilon}=u_{-\varepsilon}$ and $v_{\varepsilon}=-v_{-\varepsilon}$, the nonzero energy contribution to the right-hand side of Eq. (17) takes the form

$$
\sum_{\varepsilon_{i} \neq 0} \frac{2 v_{i}-1}{2} \Psi_{i} \Psi_{i}^{\dagger}=\sum_{\varepsilon_{i}<0}\left(\begin{array}{cc}
0, & A u_{i} v_{i}^{*} \\
v_{i} u_{i}^{*}, & 0
\end{array}\right) .
$$

On the other hand, the zero-energy eigenfunctions (9) of the clean graphene Hamiltonian reside solely on one sublattice and their contribution to the projection operator can only be a diagonal matrix

$$
\sum_{\varepsilon_{i}=0} \frac{2 v_{i}-1}{2} \Psi_{i} \Psi_{i}^{\dagger}=\sum_{\varepsilon_{i}=0} \frac{2 v_{i}-1}{2}\left(\begin{array}{cc}
u_{i} u_{i}^{*}, & 0 \\
0, & v_{i} v_{i}^{*}
\end{array}\right) .
$$

For each state $i$ here only one component $u_{i}$ or $v_{i}$ differs from zero [Eq. (9)]. Now, one easily sees that electrons with $\varepsilon=0$ interact via exchange only with other $\varepsilon=0$ electrons. On the contrary, electrons with $\varepsilon \neq 0$ interact via exchange with the states with any energy.

Equations (9) and (19) allow us to reproduce the known result $[33,34]$ for the exchange-dominated Zeeman splitting of the $n=0$ Landau level (see Appendix B)

$$
\varepsilon_{n=0}= \pm\left(\frac{e^{2}}{2 l} \sqrt{\frac{\pi}{2}}+E_{Z}\right) .
$$

This exchange renormalized Zeeman energy is of the order of the Landau level interval $\varepsilon_{B}$ since in graphene $e^{2} /\left(\hbar v_{F}\right) \sim 1$. The two signs of $\varepsilon_{n=0}$ correspond to two spin projections on the magnetic field axis. We keep the small $E_{Z}$ in Eq. (20) to compare with Eq. (21) below.

Due to their nonzero energy, the exchange interaction for the impurity-induced localized states $\Psi_{S_{ \pm}}$[Eq. (14)] is calculated completely differently, even though they contain a one-half admixture of the $n=0$ Landau level state $\psi_{0,0}$ [Eq. (9)]. In the 
large logarithm limit $L=\ln (l / d) \gg 1$, after the subtraction of the uniform energy shift due to the unity operator in Eq. (17), the impurity states $\Psi_{S_{ \pm}}$interact via exchange only with themselves via the nondiagonal density operator (18). The energies of two lowest (occupied) of these levels are

$$
\varepsilon_{S_{-}}=-\frac{\varepsilon_{B}}{2 \sqrt{L}}-\frac{e^{2}}{2 l} \sqrt{\frac{\pi}{2}} \pm E_{Z} .
$$

The first term here is the noninteracting energy [Eq. (14)], which is suppressed by the inverse square root of the large logarithm. The largest second term is the exchange energy, which occasionally turns out to be the same as the exchange energy for the $n=0$ Landau levels without impurity [Eq. (20)]. For undoped graphene, both spin/Zeeman components of the level Eq. (21) are occupied, thus forming a singlet and reducing the total spin of the quantum Hall ferromagnet state.

The Zeeman splitting of the $n=0$ Landau level enhanced by the exchange interaction [Eq. (20)] does not depend on the angular momentum quantum number $m$. This is very natural since our special choice of the vector potential, which made $m$ a good quantum number, introduces only a spurious breaking of the translational invariance in physically homogeneous systems. Adding the impurity atom breaks the translational invariance and the electron energy may now depend on angular momentum. This, however, does not happen in the noninteracting case of the previous section, where only the electrons with $m=0$ were affected by the point impurity. The actual dependence on the angular momentum appears only after taking into account the exchange interaction.

The mechanism leading to the $m$ dependence of the energy levels is also interesting and relies on the difference between zero-energy and non-zero-energy eigenstates of the particlehole-symmetric Dirac equation discussed above. As we have shown, electrons from the $n=0$ Landau level interact only with themselves via the exchange interaction. For example, any $n=0$ electron from the $K^{\prime}$ valley $\left[\psi_{0, m}^{\prime}\right.$ in Eq. (9)] interacts with the fully occupied $n=0$ level for the electron with spin down, or with the completely empty $n=0$ level for the electron with spin up. This leads to the energy Eq. (20). Note that after the subtraction of the constant energy shift due to a contact term in Eq. (17), there is a nontrivial interaction with both occupied and empty states.

On the other hand, the electron with spin down from the $K$ valley and with $m \neq 0$ does not see the exchange attraction from the same level with $m=0$, which was taken to build the finite-energy states $\Psi_{S_{ \pm}}$[Eq. (14)]. Thus, the missing level pushes up the energies of levels around it (see Fig. 4). This effect is strongest for the closest to the adatom electron with $m=1$, which becomes the easiest electron to excite. The upward shift of the levels becomes smaller with increasing angular momentum. Similarly, for the empty subband of the $n=0$ Landau level from the $K$ valley and with spin up, the absence of the level with $m=0$ in the sum for the projection operator (19) lowers the energies of the states with $m=1,2, \ldots$ Explicit calculation of the energy levels with $m \geqslant 1$, leading to

$$
\varepsilon_{0, m}= \pm \frac{e^{2} \sqrt{\pi}}{2 \sqrt{2} l}\left(1-\frac{\sqrt{2}(2 m) !}{8^{m}(m !)^{2}}\right),
$$

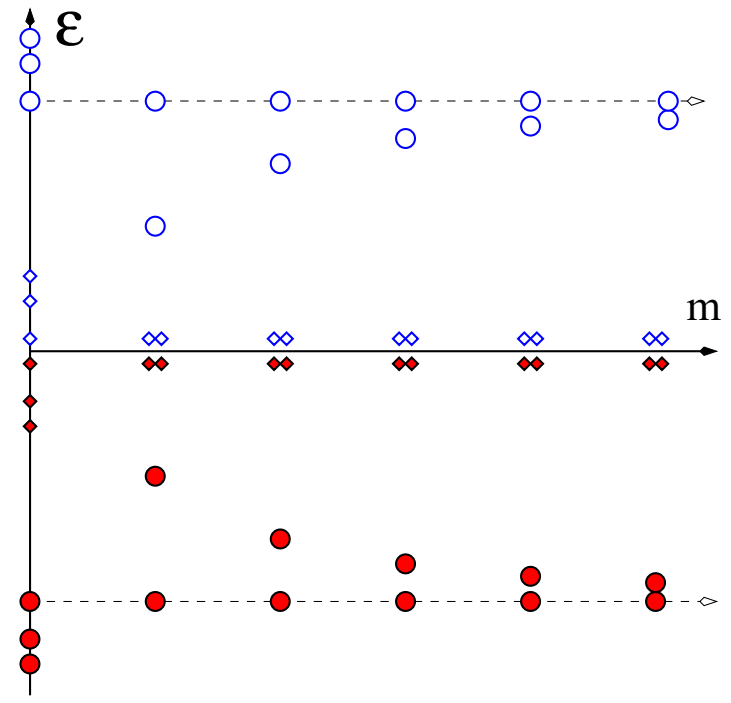

FIG. 4. (Color online) Energies (schematic) of the $n=0$ Landau level states around the impurity with and without electrons interaction. Small rhombuses: show the energy levels for the noninteracting problem, filled red rhombuses for filled and empty blue rhombuses for empty states. For each $m>0$ there are two valley degenerate states $\left(K\right.$ and $\left.K^{\prime}\right)$, each with two spin components, split due to the small Zeeman energy $\pm E_{Z}$. For $m=0$ there are total of six low-energy states. A pair of levels: $\operatorname{spin}$ split $n=0, m=0, K^{\prime}$-valley level behaves exactly the same way as its $m \neq 0$ companions. In addition, there are a pair of spin-split low-energy levels $\Psi_{S_{ \pm}}, \varepsilon_{S_{ \pm}}$ [Eq. (14)]. Both spin components of $\varepsilon_{S_{-}}$are occupied, forming a singlet. Big circles: show the energy levels for interacting electrons. Again, filled red circles show occupied states and empty blue circles show unoccupied states. States from the $K^{\prime}$ valley are not affected by the impurity independent on the value of $m$. Their energies are given by Eq. (20), which is shown by two dashed lines. Occasionally, the exchange interaction causes the same shift of the $\varepsilon_{S_{ \pm}}$levels, as it did for $n=0$ Landau level states in the absence of impurity. These states are shown by the two lowest red circles and by the two highest empty blue circles at $m=0$. The most interesting are the states with small, but finite values of the angular momentum number $m$ from the $K$ valley. Due to their reduced exchange interaction, these states fall inside the gap between the usual spin-up and -down $n=0$ Landau levels shown in Eq. (20).

is given in Appendix B. Energies of electron states at and around the impurity are depicted on Fig. 4.

\section{BUNCH OF IMPURITIES}

In this section, we consider a bunch of many closely spaced impurity atoms coupled all to the carbon atoms from the same sublattice $A$. The choice of fully sublattice-polarized bunch is motivated by Refs. [5-10], where it was shown that the Casimir interaction between impurities, caused by electrons in graphene, favors the sublattice ordering.

By closely spaced impurities we mean close compared to the Larmor radius $l$, but not as close as the carbon-carbon distance on the hexagonal lattice $d$. Instead of Eq. (11), we 
now write

$$
\left(\varepsilon-\varepsilon_{n}\right) f_{n}=\sum_{a} V_{n a} f_{a}, \quad\left(\varepsilon-\varepsilon_{a}\right) f_{a}=\sum_{n} V_{a n} f_{n} .
$$

Here, $f_{a}$ are the probability amplitudes to find the electron on the impurity $a$ and $f_{n}$ is the amplitude to find the electron in state $n$ in graphene plane [compare to Eq. (2)]. The impurity onsite energies $\varepsilon_{a}$ are assumed to be negligibly small.

In the case of one impurity we were able to choose a single state $\Psi_{n}$ [Eq. (10)] from each Landau level, coupled to the impurity by a uniform matrix element $V_{d}$ [Eq. (11)]. For several impurities, summation over $n$ in Eq. (23) includes both summation over the Landau levels and over the many individual states at each Landau level. Matrix elements $V_{a n}=$ $V_{n a}^{*}$ are now proportional to the value of the upper component of the particular electron's wave function at the carbon site coupled to the individual impurity.

Let $N \gg 1$ be the number of the impurity atoms. First, Eq. (23) expresses an infinite number of in-plane states amplitudes $f_{n}$ through the $N$ impurity amplitudes $f_{a}$ :

$$
f_{n}=\frac{1}{\varepsilon-\varepsilon_{n}} \sum_{a} V_{n a} f_{a} .
$$

The energy $\varepsilon$ and impurity amplitudes $f_{a}$ should then be found from the set of $N$ linear equations

$$
\left(\varepsilon-\varepsilon_{a}\right) f_{a}=\sum_{b} G_{a b} f_{b}, \quad G_{a b}=\sum_{n} \frac{V_{a n} V_{n b}}{\varepsilon-\varepsilon_{n}} .
$$

Equations (23) and (25) are exact. Explicit compact formula for the matrix $G_{a b}$ at low energies $\varepsilon$ is found in Eq. (C4) of Appendix C. The energies of impurity-induced states are then estimated as (each energy comes in a plus and minus pair)

$$
\varepsilon_{S}^{(1)}=\frac{\varepsilon_{B}}{2 \sqrt{L}}, \quad \varepsilon_{S}^{(2)} \sim \varepsilon_{B} \frac{\left\langle\left|\mathbf{r}_{a b}\right|\right\rangle}{l}, \quad \varepsilon_{S}^{(3)} \sim \varepsilon_{B} \frac{\left\langle\mathbf{r}_{a b}^{2}\right\rangle}{l^{2}}, \ldots
$$

Here, $L=\ln \left(\left\langle\left|\mathbf{r}_{a b}\right|\right\rangle / l\right) \gg 1,\left\langle\left|\mathbf{r}_{a b}\right|\right\rangle$ is a typical distance between adatoms in a bunch, $\left\langle\mathbf{r}_{a b}^{2}\right\rangle$ is a typical squared distance between adatoms, and so on. As we see, only one eigenvalue $\varepsilon_{S}^{(1)}$ remains (almost) the same as it was in case of a single impurity [Eq. (14)]. Each next energy is by a factor $\left\langle\left|\mathbf{r}_{a b}\right|\right\rangle / l \ll 1$ smaller than the previous one. The reason for such hierarchy of energy eigenvalues will be clear after considering the corresponding wave functions.

Equations (12) and (24) show that the electron wave function in graphene plane in case of multiple impurities is a superposition of $N$ single-impurity solutions $\Psi_{S}$ [Eq. (14)]. Since the impurities are very close, the resulting sum of the wave functions may differ strongly (and is different due to the severe cancellations) from any individual contribution. Among $N$ wave functions described by Eq. (25) only one having the largest energy looks close to the single-impurity solution (14) [see Eq. (C13)]. Wave functions of other impurity-induced states, having smaller energies $\varepsilon^{(2)}, \varepsilon^{(3)}$, etc., in Eq. (26), far away from the small bunch of impurities have the form

$$
\Psi_{S}^{(m)}=\left(\begin{array}{c}
e^{i \mathbf{K r} \frac{\phi_{0, m}}{\sqrt{2}}} \\
\pm e^{i \alpha \frac{e^{i \mathbf{K}^{\prime} \mathbf{r}}}{z^{*}} \frac{e^{-z z^{*} / 4}}{2 \sqrt{\pi L}}}
\end{array}\right)
$$

Here, $\alpha$ is some unknown unimportant constant. Details of calculation of $\Psi_{S}^{(m)}$ are given in Appendix C.

The accuracy of the wave function (27) is much better than the accuracy of the individual energies (26). The energies $\varepsilon_{S}^{(m)}$ are known only by the order of magnitude. The overall normalization of the components of Eq. (27) is found with $\sim 1 / \log$ accuracy, but the accuracy of, e.g., the upper component itself is much better, being determined by the small ratio of the size of the bunch and the Larmor radius.

The lower components of the wave functions $\Psi_{S}^{(m)}$ [Eq. (27)] are similar for any $m$. As was already mentioned, Eq. (27) appears as a result of strong cancellations between the single-impurity solutions generated by very close impurities. This cancellation suppresses the upper component of $\Psi_{S}^{(m)}$, but also a part of the lower component oscillating in unison with the upper one $\propto e^{i \mathbf{K r}}$. The part of the lower component behaving like $e^{i \mathbf{K}^{\prime} \mathbf{r}} / z^{*}$ survives and is shown in Eq. (27).

More interesting is the upper component of the impurityinduced state $\Psi_{S}^{(m)}$. As was discussed in Sec. II, effectively the impurity state $\Psi_{S}$ [Eq. (14)] is a superposition of the $n=0$ Landau level state $\psi_{0,0}$ [Eq. (9)] and the $\sim 1 / z$ localized state of Ref. [4]. Each of them has only one nonvanishing component, up or down. Consequently, the upper component of the $N$-impurities solution $\Psi_{S}^{(m)}$ represents a superposition of $N$ such zeroth Landau level solutions, each centered at individual impurity. However, these spatially slightly offset eigenfunctions of the graphene Hamiltonian in the magnetic field are very similar. As is shown in Appendix C, the upper components of the true solutions of the many impurities problem [Eq. (25)] became the orthogonalized combinations of the $n=0$ Landau level states generated by the individual impurities and these new orthogonal states have an (almost) well-defined value of the angular momentum quantum number $m$. It is not surprising that the states $\Psi_{S}^{(m)}$ [Eq. (27)] with large $m$ have small energies [Eq. (26)] since their coupling to the impurity atom upper component vanishes at small distances as $\phi_{0, m} \sim z^{* m}$.

Electrons described by Eq. (27) have a probability $\frac{1}{2}$ to be found in the $n=0$ Landau level state in the $K$ valley. To preserve the electroneutrality of undoped graphene, each such $n=0$ state should be on average occupied by a one electron, which is achieved if both spin components of the negative-energy states $\Psi_{S}^{(m)}$ [Eq. (27)] are occupied. Thus, we expect the electrons from the $K$ valley $n=0$ Landau level surrounding bunch of impurities to form a large (radius $R=$ $\sqrt{2 N} l$ ) unpolarized droplet inside the spin-polarized quantum Hall ferromagnet, as is shown in Fig. 5. The $n=0$ Landau level electrons from the $K^{\prime}$ valley remain fully spin polarized.

\section{A. Many impurities with interaction}

As was shown in Sec. III, energies of electrons surrounding the single-impurity atom are strongly modified by the electronelectron interaction. The same is true in case of multiple impurities. The simple calculation sketched in Appendix D gives the energies of impurity-induced states (27):

$$
\varepsilon_{S}^{(m)}= \pm \frac{(2 m) !}{(m !)^{2} 2^{2 m}} \sqrt{\frac{\pi}{2}} \frac{e^{2}}{2 l} .
$$




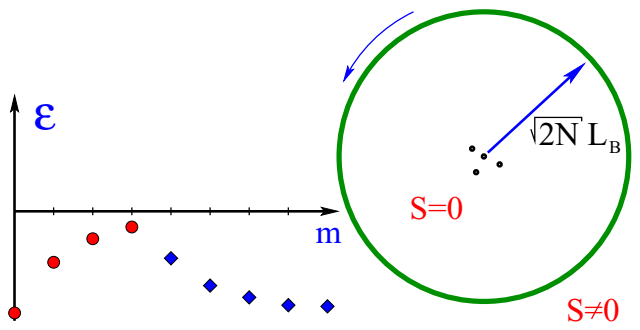

FIG. 5. (Color online) Right: a large droplet of reduced spin polarization surrounding a bunch of impurities. Left: schematic angular momentum dependence of the energy of impurity-induced states (red circles) and the energy of the $n=0$ sublattice $A$ electron states surrounding the droplet (blue rhombuses). Due to their nonuniform exchange energy, these latter states carry an edge current around the droplet.

Unlike the noninteracting result (26), all energies (28) are of the same formal order of magnitude $\sim e^{2} / l$. The decrease of $\varepsilon_{S}^{(m)}$ with increasing $m$ is now due to a pure numerical factor. Although the electronic interaction renormalizes strongly the energy eigenvalues, wave functions (27) are intact and determine the dynamics of electrons surrounding the bunch of adatoms.

The particle-hole symmetry ensures that the occupied electron states $\Psi_{S}^{(m)}$ [Eq. (27)] with $0 \leqslant m<N$ and the Landau level states $\psi_{0, m}$ [Eq. (9)] with $m \geqslant N$ create a constant charge density around the bunch of adatoms. This means that the direct electrons interaction is trivial. However, as follows from Eqs. (15), (18), and (19), the states $\Psi_{S}^{(m)}$ and $\psi_{0, m}$ do not interact via the exchange interaction. Consequently, the electrons with $m>N$ see the weaker exchange interaction since they are missing the interaction with the electrons from inside the circle $r<l \sqrt{2 N}$. Energies of these states, occupied or empty, may be written as

$$
\varepsilon_{0, m>N}=\mp \frac{e^{2}}{2 l} \sqrt{\frac{\pi}{2}} g(m-N),
$$

where the function $g(x)$ increases smoothly from $g(0)=\frac{1}{2}$ to $g(x \gg \sqrt{N})=1$. Explicit form of $g(x)$ could not be found in a compact form, but some steps towards its evaluation are given in Appendix D.

The electron density for each state $\psi_{0, m}$ has a form of the narrow ring with the radius $R_{m}=l \sqrt{2 m}$. The $m$ dependence of the energies $\varepsilon_{0, m>N}$ [Eq. (29)] of these ring states means that there is an edge state carrying a persistent current around the spinless electron droplet.

\section{v. CONCLUSIONS}

The aim of this paper was to investigate the effect of resonant adatom impurities on the spectrum and electronic wave functions in graphene in quantizing magnetic field. Despite the broad interest in properties of graphene with resonant impurities [5-19], almost nobody up to now (except for Ref. [19] in our list) has considered their role in the quantum Hall regime. This gap needed to be closed simply because of the experiments in magnetic field are much easier in graphene than in other two-dimensional materials $[1,2]$. Luckily, we also found several interesting effects, especially related to the electrons' interaction, which may potentially be observed in functionalized graphene in this regime.

The resonance condition requires the impurity atom having an electron level with the energy very close to the Dirac point in graphene and the belief in the existence of such impurities is based on the theoretical calculations of Refs. [12-14]. Experimental confirmation of these predictions, or even a direct search for the materials having the proper resonant levels is thus highly desired. Consequently, the direct measurement of the spectrum of electrons surrounding the impurity [Eq. (22)] may serve as a proof of the resonance. The apparatus necessary for such experiments was already developed in Ref. [35], where the redistribution of the Landau levels occupation around the charged impurity was measured with the scanning tunneling microscope.

One may also consider the same model with the impurity energy $\varepsilon_{d}$ shifted away from the neutrality point. The symmetry between the positive- and negative-energy solutions of the Dirac equation will then be broken, leading to the in-plane charge redistribution and broken charge neutrality. The induced charges, however, will create the electrostatic potential pushing the impurity level back to the Dirac point. Investigation of such self-consistent stabilization of the model (1) with zero $\varepsilon_{d}$ is an interesting problem for a future investigation.

In this paper, we were able to successfully describe not only graphene with a single impurity, but also made a considerable progress in solving the problem with many impurities forming a small bunch. Such a strongly inhomogeneous distribution of atomic impurities, which was never considered theoretically, is the (likely) possible outcome of experimental functionalization of graphene. According to our prediction, the bunch of impurities creates a large droplet of reduced spin inside a quantum Hall ferromagnet, encircled by a current carrying edge state. Similarly to the electron states induced by the single impurity, such droplets should be measurable by the scanning tunneling microscope. In addition, due to its large size, even a single such droplet may affect the transport in graphene mesoscopic devices.

\section{ACKNOWLEDGMENTS}

Discussions with P. W. Brouwer, E. Kandelaki, P. Recher, M. Schneider, G. Schwiete, and L. Weithofer are greatly appreciated. The author acknowledges hospitality of the Institute for Advanced Studies at the Hebrew University, Jerusalem. This work was supported by the Alexander von Humboldt Foundation and by the DFG Grant No. RE 2978/1-1.

\section{APPENDIX A: SOLVING THE DIRAC EQUATION WITH A SINGLE IMPURITY}

In this Appendix we present the solution of Eq. (12) for the energy of the impurity-induced localized state in magnetic field. We will then find the explicit shape of the localized state in the coordinate representation Eq. (14).

Impurity-induced localized states, which are the solutions of Eq. (12) most close to the Dirac point, have $|\varepsilon| \ll \varepsilon_{B}$. Therefore, it is convenient to consider separately the $n=0$ 
and $n \neq 0$ contributions to the sum in the right-hand side of Eq. (12). For $n \neq 0$, the contributions with $n>0$ and $n<0$ almost cancel each other. It is enough to consider the result of this cancellation in the linear in $\varepsilon$ approximation. With the energies $\varepsilon_{n}$ found in Eq. (8) one has

$$
\varepsilon-\varepsilon_{d}=\frac{V_{d}^{2}}{\varepsilon}-\sum_{n=1}^{n_{\max }} \frac{2 \varepsilon V_{d}^{2}}{\varepsilon_{n}^{2}-\varepsilon^{2}} \approx \frac{V_{d}^{2}}{\varepsilon}-\varepsilon \frac{V_{d}^{2}}{\varepsilon_{B}^{2}} \sum_{n=1}^{n_{\max }} \frac{2}{n} .
$$

The sum over $n$ here diverges only logarithmically and in the large logarithm approximation it is enough to get only a rough estimate of the upper cutoff $n_{\max }$. Assuming that the solutions of the continuous Dirac equation (8) may be used only for energies small compared to the bandwidth $(\sim t)$ gives $\sqrt{n_{\max }} \varepsilon_{B} \approx t$, or

$$
n_{\max } \approx(l / d)^{2}
$$

The carbon-to-impurity coupling and the carbon-carbon hopping matrix element are expected to be of the same order of magnitude $U \sim t$. This means that in the large logarithm approximation, the left-hand side of Eq. (A1) should be neglected and the energies of two impurity-induced levels become

$$
\varepsilon_{S_{ \pm}}= \pm \frac{\varepsilon_{B}}{2 \sqrt{L}}
$$

where $L=\ln (l / d) \gg 1$. In the leading approximation, the two energies $\varepsilon_{S_{ \pm}}$do not depend on the strength of the coupling to the impurity $U$.

The normalization condition (13) gives the probability for the electron to stay at the impurity atom

$$
f_{d \pm}^{2} \approx \frac{\sqrt{3} \pi}{4} \frac{t^{2}}{U^{2} L}
$$

Since $U / t \sim 1$, the probability to find electron at the adatom is small like the inverse of a large logarithm.

Now, one may find explicitly the impurity-induced state wave function (14)

$$
\Psi_{S_{ \pm}}=\sum f_{n} \Psi_{n}
$$

using the Landau level states $\Psi_{n}$ coupled to the impurity [Eq. (10)] and the amplitudes $f_{n}=V_{d} f_{d} /\left(\varepsilon-\varepsilon_{n}\right)$ [Eq. (12)]. In the leading approximation, the energy $\varepsilon$ in the denominator in $f_{n} \sim 1 /\left(\varepsilon-\varepsilon_{n}\right)$ may be neglected for all $n \neq 0$. After that, the contribution to the upper component of $\Psi_{S_{ \pm}}$from the Landau level states with $n \neq 0$ vanishes, leading to

$$
\begin{aligned}
\Psi_{S_{ \pm}} \sim & \frac{e^{i \mathbf{K r}}}{\varepsilon_{S_{ \pm}}}\left(\begin{array}{c}
\phi_{0,0} \\
0
\end{array}\right) \\
& +\sum_{n=1}^{n_{\max }} \frac{i}{\varepsilon_{B} \sqrt{n}}\left(\begin{array}{c}
0 \\
e^{i \mathbf{K} \mathbf{r}} \phi_{n-1,1}+e^{i \mathbf{K}^{\prime} \mathbf{r}} \phi_{n,-1}
\end{array}\right) .
\end{aligned}
$$

This formula should be compared with the final result for the wave function [Eq. (14)]. At first sight, it seems surprising: How the lower component of the impurity state (14) may be singular at small distances, if it is built from the functions $\phi_{n-1,1}$ and $\phi_{n,-1}$, which all vanish at $z \rightarrow 0$ ? To understand this, let us consider the small-distance behavior of these functions

$$
\phi_{n-1,1} \approx \sqrt{\frac{n}{4 \pi}} z^{*}\left(1-\frac{n|z|^{2}}{2}+\cdots\right)
$$

and

$$
\phi_{n,-1} \approx-\sqrt{\frac{n}{4 \pi}} z\left(1-\frac{n|z|^{2}}{2}+\cdots\right) .
$$

For large $n$ even near the origin both $\phi_{n-1,1}$ and $\phi_{n,-1}$ are oscillating functions of $|z|$. We keep the second term of the expansion at small $|z|$ in Eqs. (A7) and (A8) to show that the period of these oscillations scales like $\Delta|z| \sim 1 / \sqrt{n}$. Thus, the short-distance behavior, for example, of a first sum in Eq. (A6) is

$$
\sum_{n} \frac{1}{\sqrt{n}} \phi_{n-1,1} \sim z^{*} \int_{0}^{1 /|z|^{2}} d n \sim \frac{1}{z} .
$$

This simple estimate does not yet allow us to find an overall numerical factor at the $1 / z^{*}(1 / z)$ term. This factor may be found from the condition of orthogonality of $\Psi_{S_{+}}$and $\Psi_{S_{-}}$, leading to Eq. (14).

More complicated is finding the enveloping function $e^{-|z|^{2} / 4}$ of the lower component of $\Psi_{S_{ \pm}}$in Eq. (14). Derivation of this long-distance behavior is given in the following.

\section{Large-distance asymptotics of the singular state}

First, let us write the spinor wave function (A6) in a form $\Psi_{S}=e^{i \mathbf{K r}} \psi+e^{i \mathbf{K}^{\prime} \mathbf{r}} \psi^{\prime}$, where the two smooth spinor functions have a form

$$
\psi=\left(\begin{array}{c}
u(r) \\
i e^{-i \phi} v(r)
\end{array}\right), \quad \psi^{\prime}=\left(\begin{array}{c}
\tilde{u}(r) \\
i e^{i \phi} \tilde{v}(r)
\end{array}\right),
$$

and we introduced the polar coordinates $x=r \cos \phi, y=$ $r \sin \phi$. The four functions $u, v, \tilde{u}, \tilde{v}$ satisfy two systems of equations

$$
\frac{\varepsilon}{\sqrt{2} \varepsilon_{B}} v=\frac{d u}{d r}+\frac{1}{2} r u, \quad \frac{\varepsilon}{\sqrt{2} \varepsilon_{B}} u=-\frac{d v}{d r}-\frac{v}{r}+\frac{1}{2} r v
$$

and

$$
\frac{\varepsilon}{\sqrt{2} \varepsilon_{B}} \tilde{v}=-\frac{d \tilde{u}}{d r}+\frac{1}{2} r \tilde{u}, \quad \frac{\varepsilon}{\sqrt{2} \varepsilon_{B}} \tilde{u}=\frac{d \tilde{v}}{d r}+\frac{\tilde{v}}{r}+\frac{1}{2} r \tilde{v} .
$$

Since the energy $\varepsilon \ll \varepsilon_{B}$ is small, one may solve these equations iteratively: First find the solution for $\varepsilon=0$ and then look for the corrections $\sim \varepsilon, \sim \varepsilon^{2}$, etc.

For $\varepsilon=0$, Eqs. (A11) and (A12) become a set of decoupled first-order differential equations, leading to four independent (in general non-normalizable) solutions

$$
\begin{aligned}
& \text { (1) } u=e^{-r^{2} / 4}, \quad v=\tilde{u}=\tilde{v}=0, \\
& \text { (2) } v=\frac{1}{r} e^{r^{2} / 4}, \quad u=\tilde{u}=\tilde{v}=0, \\
& \text { (3) } \tilde{u}=e^{r^{2} / 4}, \quad v=u=\tilde{v}=0, \\
& \text { (4) } \tilde{v}=\frac{1}{r} e^{-r^{2} / 4}, \quad v=u=\tilde{u}=0 .
\end{aligned}
$$


Using only the zero-energy solutions which are regular at large distances 1 and 4, we find three of the functions introduced in Eq. (A10)

$$
u=e^{-\rho^{2} / 4}, \quad \tilde{u}=0, \quad \tilde{v}=\frac{c}{\rho} e^{-\rho^{2} / 4},
$$

where the coefficient $c$ is found from Eq. (14).

To find the last function $v$, we take finite energy $\varepsilon$ and substitute $u$ [Eq. (A14)] into the second equation (A11),

$$
v=\frac{\varepsilon l_{B}}{\hbar v_{F}} \frac{1}{\rho} e^{\rho^{2} / 4} \int_{\rho}^{\infty} x e^{-x^{2} / 2} d x=\frac{\varepsilon l_{B}}{\hbar v_{F}} \frac{e^{-\rho^{2} / 4}}{\rho} .
$$

\section{APPENDIX B: CALCULATING THE EXCHANGE ENERGY}

This section describes the derivation of the exchange interaction matrix elements presented in the main text. Using Eqs. (9) and (19), the exchange energy of the electron with the orbital quantum number $M$ is written in the form

$$
\begin{aligned}
\varepsilon_{M}= & \mp \sum_{m=0}^{\infty} \int \frac{e^{2}}{2\left|\mathbf{r}-\mathbf{r}^{\prime}\right|} \phi_{0, M}^{*}(\mathbf{r}) \phi_{0, m}(\mathbf{r}) \\
& \times \phi_{0, m}^{*}\left(\mathbf{r}^{\prime}\right) \phi_{0, M}\left(\mathbf{r}^{\prime}\right) d \mathbf{r} d \mathbf{r}^{\prime} .
\end{aligned}
$$

The energy is negative for occupied states and positive for empty ones. Summation over $m$ in Eq. (B1) gives the projection operator onto the occupied $n=0$ Landau level, which may be found exactly

$$
\begin{aligned}
\rho\left(\mathbf{r}, \mathbf{r}^{\prime}\right) & =\sum_{m=0}^{\infty} \phi_{0, m}(\mathbf{r}) \phi_{0, m}^{*}\left(\mathbf{r}^{\prime}\right) \\
& =\sum_{m=0}^{\infty} \frac{\left(z^{*} z^{\prime} / 2\right)^{m}}{2 \pi m !} e^{-|z|^{2} / 4-\left|z^{\prime}\right|^{2} / 4} \\
& =\frac{1}{2 \pi} \exp \left\{-\frac{|z|^{2}+\left|z^{\prime}\right|^{2}-2 z^{*} z^{\prime}}{4}\right\} \\
& =\frac{1}{2 \pi} \exp \left\{-\frac{\left|z-z^{\prime}\right|^{2}}{4}+\frac{z^{*} z^{\prime}-z z^{\prime *}}{4}\right\} .
\end{aligned}
$$

Note that the second term in the argument of the exponent in the last line here is pure imaginary (a phase). The energies are found from the generating function $I(\alpha)$,

$$
\varepsilon_{M}=\left.\mp \frac{1}{M !}\left(\frac{\partial}{\partial \alpha}\right)^{M} I(\alpha)\right|_{\alpha=0},
$$

where

$$
\begin{aligned}
I(\alpha)= & \int \frac{e^{2}}{2 l\left|z-z^{\prime}\right|} \frac{d^{2} z d^{2} z^{\prime}}{4 \pi^{2}} \\
& \times \exp \left\{-\frac{|z|^{2}+\left|z^{\prime}\right|^{2}-z^{*} z^{\prime}-\alpha z z^{\prime *}}{2}\right\} .
\end{aligned}
$$

Introducing new variables $u=z-z^{\prime}, v=z+z^{\prime}$ one finds

$$
I(\alpha)=\frac{e^{2} \sqrt{\pi}}{2 \sqrt{2}(1-\alpha) l},
$$

leading to Eq. (20).

Exchange-dominated Zeeman splitting of the singular impurity level (14) is calculated with the help of the density operator (18). The exchange energy in this case is dominated by the interaction of the impurity level with itself,

$$
\varepsilon_{S}=\mp 2 \int \frac{e^{2}}{\left|\mathbf{r}-\mathbf{r}^{\prime}\right|}\left|\Psi_{S_{1}}(\mathbf{r})\right|^{2}\left|\Psi_{S_{2}}\left(\mathbf{r}^{\prime}\right)\right|^{2} d \mathbf{r} d \mathbf{r}^{\prime} .
$$

Here, $\Psi_{S_{1}}$ and $\Psi_{S_{2}}$ are the upper and lower components of the spinor function $\Psi_{S}$ [Eq. (14)]. The energy [Eq. (B6)] formally coincides with the Coulomb interaction energy of two charge densities $\left|\Psi_{S_{1}}(\mathbf{r})\right|^{2}$ and $\left|\Psi_{S_{2}}\left(\mathbf{r}^{\prime}\right)\right|^{2}$. The calculation of the energy is greatly simplified after one notices that the density $\left|\Psi_{S_{2}}\left(\mathbf{r}^{\prime}\right)\right|^{2}$ is mostly concentrated at distances small compared to the Larmor radius $r \ll l$, and the total charge in each component of the wave function is $\frac{1}{2}$. Thus, for $\ln (l / d) \gg 1$,

$$
\varepsilon_{S}=\mp \int \frac{e^{2}}{r}\left|\Psi_{S_{1}}(\mathbf{r})\right|^{2} d \mathbf{r}=\mp \frac{e^{2}}{2 l} \sqrt{\frac{\pi}{2}} .
$$

The exchange-dominated splitting of $n=0$ Landau level [Eq. (20)] does not depend on the angular quantum number $M$, as it should be for the translationally invariant system. The $M$ dependence appears in the case of impurity adatom considered in this paper. As was discussed in the main text of the paper, the electron states with $M \neq 0$ from the $n=0$ Landau level effectively know about the impurity because of missing the exchange interaction with the $n=0, m=0$ electron. Corresponding correction to the energy is given by the same formula (B1), where one leaves only the $m=0$ term in the sum, i.e.,

$$
\begin{aligned}
\Delta \varepsilon_{M}= & \pm\left.\frac{1}{M !}\left(\frac{\partial}{\partial \alpha}\right)^{M} J(\alpha)\right|_{\alpha=0}, \\
J(\alpha)= & \int \frac{e^{2}}{2 l\left|z-z^{\prime}\right|} \frac{d^{2} z d^{2} z^{\prime}}{4 \pi^{2}} \\
& \times \exp \left\{-\frac{|z|^{2}+\left|z^{\prime}\right|^{2}-\alpha z z^{\prime *}}{2}\right\} \\
= & \frac{e^{2}}{4 l} \sqrt{\frac{2 \pi}{2-\alpha}} .
\end{aligned}
$$

This leads to Eq. (22).

\section{APPENDIX C: SOLVING THE DIRAC EQUATION WITH SEVERAL IMPURITIES}

In order to find the energies of impurity-induced states from Eq. (25) one needs to know the matrix $G_{a b}$, describing the electron's hopping between the impurity sites. In the case of an atom placed at the origin $z_{a}=\left(x_{a}+i y_{a}\right) / l=0$, and for the vector potential in a polar gauge $\mathbf{A}=(B y / 2,-B x / 2,0)$, the matrix element $G_{a a}$ was already found in Eq. (A1):

$$
G_{a a}=\frac{V_{d}^{2}}{\varepsilon}\left[1-4 \frac{\varepsilon^{2}}{\varepsilon_{B}^{2}} \ln \frac{l_{B}}{d}\right] .
$$

We will see in a moment that this formula works for any diagonal element of the matrix $G_{a b}$.

Equation $(\mathrm{C} 1)$ is valid in the limit $\ln (l / d) \gg 1$. To reach the better accuracy, one would need to go beyond the Dirac equation approximation and to find the electron wave functions on the hexagonal lattice in magnetic field. Consequently, corrections of higher orders in small energy $\sim \varepsilon^{3}$ are also 
neglected in Eq. (C1). [The same holds for the accuracy of Eqs. (C2) and (C4).]

Suppose now that the atom $b$ is placed at the origin $\left(z_{b}=0\right)$ and the atom $a$ is not. Calculation of the element of the matrix $G_{a b}$ now formally coincides with the calculation of the upper component of the singular impurity state wave function (A6), which gives

$$
G_{a b}=\frac{V_{d}^{2}}{\varepsilon}\left[1-4 \frac{\varepsilon^{2}}{\varepsilon_{B}^{2}} \ln \frac{1}{\left|z_{a b}\right|}\right] e^{-\left|z_{a b}\right|^{2} / 4} .
$$

Here, $z_{a b}=z_{a}-z_{b}$ and $\left|z_{a b}\right|=r_{a b} / l$, where $r_{a b}$ is the true distance between the atoms $a$ and $b$.

The value of the matrix element $G_{a b}$ [Eq. (C2)] depends only on the distance between two atoms $\left|z_{a b}\right|$. Thus, one is tempted to use this formula in case of arbitrary positions of both atoms. However, for the equation (C2) to be valid, it is also important that the vector potential $\mathbf{A}$ also vanishes at the position of one of the atoms $a$ or $b$. The shift of the position of vanishing of (the both components of) the vector potential is achieved by the simple gauge transformation, adding a factor

$$
\exp \left\{i \frac{x_{a} y_{b}-y_{a} x_{b}}{2 l^{2}}\right\}
$$

to the equation (C2). After taking into account this phase, the formula for the element of the matrix may be written in a simple form

$$
\begin{aligned}
G_{a b}= & \frac{V_{d}^{2}}{\varepsilon}\left[1-4 \frac{\varepsilon^{2}}{\varepsilon_{B}^{2}} \ln \frac{1}{\left|z_{a b}\right|}\right] \\
& \times \exp \left\{-\frac{\left|z_{a}\right|^{2}+\left|z_{b}\right|^{2}-2 z_{a} z_{b}^{*}}{4}\right\},
\end{aligned}
$$

where now the "coordinates" $z_{a}$ and $z_{b}$ are measured from the point of vanishing of the vector potential, which is chosen to be somewhere in the middle of the bunch of impurities.

It is convenient to introduce the new variables $\phi_{a}$,

$$
\phi_{a}=e^{-\left|z_{a}\right|^{2} / 4} f_{a}
$$

and rewrite Eq. (25) in a form

$$
\begin{aligned}
(\varepsilon & \left.-\varepsilon_{a}\right) e^{\left|z_{a}\right|^{2} / 2} \phi_{a} \\
& =\frac{V_{d}^{2}}{\varepsilon} \sum_{b}\left[1-4 \frac{\varepsilon^{2}}{\varepsilon_{B}^{2}} \ln \frac{1}{\left|z_{a b}\right|}\right] \exp \left\{\frac{z_{a}^{*} z_{b}}{2}\right\} \phi_{b} .
\end{aligned}
$$

In the large logarithm approximation since $\varepsilon_{a} \ll \varepsilon_{B}$ and $V_{d} \sim \varepsilon_{B}$, the left-hand side of this equation should be omitted, leading to

$$
\sum_{b} \exp \left\{\frac{z_{a}^{*} z_{b}}{2}\right\} \phi_{b}=4 \frac{\varepsilon^{2}}{\varepsilon_{B}^{2}} \sum_{b} \ln \frac{1}{\left|z_{a b}\right|} \exp \left\{\frac{z_{a}^{*} z_{b}}{2}\right\} \phi_{b} .
$$

This is the final equation, written in the most compact form, which one needs to solve in order to find the energies and wave functions of the impurity-induced localized states. There is certain freedom in the choice of positions of the origin $z=0$, which may be (but do not necessarily have to) fixed by, e.g., requiring vanishing of the average distance $\sum_{a} z_{a}=0$.

Equation (C7) is an eigenvalue problem of the form $A \phi=\varepsilon^{2} B \phi$, where $A$ and $B$ are two Hermitian matrices. What makes this problem tractable is that in case of all $\left|z_{a}\right| \ll 1$, the matrix in the left-hand side of the equation $A_{a b}=e^{z_{a}^{*} z_{b} / 2}$ has eigenvalues of a very different magnitude. Indeed, one may expand the exponent $e^{z_{a}^{*} z_{b} / 2}$ in a power series and find (for a bunch of impurities centered as $\sum z_{a}=0$ ) the three first largest eigenvalues $N, \frac{1}{2} \sum\left|z_{a}\right|^{2}$, and $\frac{1}{8}\left[\sum\left|z_{a}\right|^{4}-\right.$ $\left.\frac{1}{N}\left|\sum z_{a}^{2}\right|^{2}-\left.\left.\left|\sum z_{a}\right| z_{a}\right|^{2}\right|^{2} /\left(\sum\left|z_{a}\right|^{2}\right)\right]$. Each next eigenvalue (having more and more complicated explicit form) will contain higher powers of small $\left|z_{a}\right|^{2}$.

We will show now that this property of the matrix $e^{z_{a}^{*} z_{b} / 2}$ leads to a hierarchical structure of the eigenvalues of Eq. (25):

$$
\left|\varepsilon^{(1)}\right| \gg\left|\varepsilon^{(2)}\right| \gg\left|\varepsilon^{(3)}\right| \cdots \text {. }
$$

We use superscript indices to enumerate the energies $\varepsilon^{(i)}$ to avoid confusion with the Landau level and impurity atom energies $\varepsilon_{n}, \varepsilon_{a}$. As always, in the particle-hole-symmetric limit, $\varepsilon_{a} \rightarrow 0$, the eigenvalues appear in \pm pairs, as is obvious from Eq. (C7).

To investigate the properties of the solutions of Eq. (C7), let us consider the series of several consecutive approximations to this equation.

First iteration. At first step, we neglect completely all the small arguments in the exponent $e^{z_{a}^{*} z_{b} / 2}$ both in the left- and right-hand sides of Eq. (C7), leading to

$$
\sum_{b} \phi_{b}=4 \frac{\varepsilon^{2}}{\varepsilon_{B}^{2}} \sum_{b} \ln \frac{1}{\left|z_{a b}\right|} \phi_{b} .
$$

Remarkably, this equation has $N-1$ exact zero-energy solutions $\phi_{a}^{(i)}$, satisfying a single constraint

$$
\sum_{b} \phi_{b}^{(i)}=0, \quad \varepsilon^{(i)} \equiv 0 .
$$

The last and only nontrivial solution in the limit of all large logarithms has the energy

$$
\varepsilon \approx \pm \frac{\varepsilon_{B}}{2 \sqrt{\ln \left(1 /\left\langle\left|z_{a b}\right|\right\rangle\right)}},
$$

where $\left\langle\left|z_{a b}\right|\right\rangle$ is some average distance between impurities. The corresponding eigenfunction has a simple form

$$
\phi_{a} \approx 1 / \sqrt{N},
$$

only if not only all the logarithms in the right-hand side of Eq. (C9) are large, but also if there are only two different large $\operatorname{logarithms} L_{1}=\ln (a / d)$ (in case $\left.a=b\right)$ and $L_{2}=\ln \left(1 /\left|z_{a b}\right|\right)$ (in case $a \neq b$ ). This means that the distance between any two impurities in the bunch is of the same (close) order of magnitude.

Each amplitude $\phi_{a}$ via Eqs. (C5) and (24) generates a contribution of the form (14) to the electrons wave function in graphene, centered at the impurity $a$. Even though Eqs. (C11) and (C12) have a rather poor (at best $\sim 1 / \log$ ) accuracy, they are enough to make strong conclusions about the wave function of the largest energy impurity state. The upper component of the wave function consists of the sum of many $m=0$ functions with slightly offset centers and slightly different phases [as in Eq. (C3)], which however due to $\left|z_{a}\right| \ll 1$ form an almost unperturbed joint $m=0$ state. The lower component consists of many singular $\sim 1 / z$ states [4] centered at individual adatoms in the lower component. Exactly as it was in the case of single adatom, due to the orthogonality of "+" and "-_" 
states [Eq. (C11)], both of them should have equal probability to find electron in upper and lower components. Outside the compact bunch of impurities, the electron wave function for the highest-energy impurity state has the form [compare to Eq. (14)]

$$
\Psi_{m=0}=\left(\begin{array}{c}
e^{i \mathbf{K r} \frac{e^{-z z^{*} / 4}}{2 \sqrt{\pi}}} \\
\pm\left(\alpha \frac{e^{i \mathbf{K r}}}{z}+\beta \frac{e^{i \mathbf{K}^{\prime} \mathbf{r}}}{z^{*}}\right) \frac{e^{-z z^{*} / 4}}{2 \sqrt{\pi L}}
\end{array}\right) .
$$

Here, $\alpha$ and $\beta$ are two complex numbers, $|\alpha|^{2}+|\beta|^{2}=1$, and we remind that $\mathbf{K}^{\prime}=-\mathbf{K}$. Also, the large logarithm here is $L=$ $\ln \left(1 /\left\langle\left|z_{a b}\right|\right\rangle\right) \gg 1$, where $\left\langle\left|z_{a b}\right|\right\rangle \ll 1$ is the typical distance between impurities.

Even more interesting are the other $N-1$ low-energy states described by Eq. (C10). The constraint $\sum_{b} \phi_{b}^{(i)}=0$ means that the amplitude of $n=0, m=0$ states in their upper component (almost) vanish. Thus, these states are the superpositions of other than $m=0$ states in the upper component (i.e., $m=$ $1,2, \ldots, N-1)$.

Second iteration. Our next step will be to expand the exponent $e^{z_{a}^{*} z_{b} / 2}$ in the left-hand side of Eq. (C7) to the first order in a small argument $z_{a}^{*} z_{b} / 2$, leading to

$$
\sum_{b} \phi_{b}+\frac{z_{a}^{*}}{2} \sum_{b} z_{b} \phi_{b}=4 \frac{\varepsilon^{2}}{\varepsilon_{B}^{2}} \sum_{b} \ln \frac{1}{\left|z_{a b}\right|} \phi_{b} .
$$

Keeping the same $\sim z_{a}^{*} z_{b}$ terms in the right-hand side of Eq. (C7) would not add any new qualitative features to the solution.

Instead of Eq. (C10), now any vector $\phi_{a}^{(i)}$ satisfying two simple constraints would be an exact zero-energy solution of the system of equations (C14):

$$
\sum_{b} \phi_{b}^{(i)}=0, \quad \sum_{b} z_{b} \phi_{b}^{(i)}=0, \quad \varepsilon^{(i)} \equiv 0 .
$$

Thus, there are $N-2$ exact zero-energy solutions.

Among the remaining two nontrivial solutions one, with the larger energy, was described by Eqs. (C11) and (C12). The second solution for a center of the bunch chosen to satisfy $\sum z_{a}=0$ has the form

$$
\phi_{a} \sim z_{a}^{*}, \quad \varepsilon \approx \pm \varepsilon_{B} \sqrt{\frac{\sum\left|z_{b}\right|^{2}}{8\left(L_{1}-L_{2}\right)}},
$$

where $L_{1}$ and $L_{2}$ are two large logarithms defined below Eq. (C12).

One may continue expanding the matrix exponent $e^{z_{a}^{*} z_{b} / 2}$ in the left-hand side of Eq. (C7) to higher orders in the small argument, to find the finite values of smaller and smaller eigenvalues. The resulting estimate of the energy values is given by Eq. (26). The wave function for the $m$ th state outside the small bunch of impurities is given by Eq. (27). As we told, the in-plane wave function for many impurities is built as a sum of single-impurity solutions (14), centered at individual impurities and with a gauge factor accounting for the center displacement. Since for $m>0$ these single-impurity contributions strongly cancel each other, there is no $\sim e^{i \mathbf{K r}} / z$ contribution in the lower component of Eq. (14). Contributions $\sim e^{i \mathbf{K}^{\prime} \mathbf{r}} / z^{*}$ in the lower component of the pseudospinor come with the phase which is not synchronized with the phase of the the upper component and thus are not suppressed. The upper components of different impurity states each acquire an (almost) well-defined and different value of the angular momentum $m$.

\section{APPENDIX D: EXCHANGE INTERACTION IN CASE OF MANY ADATOMS}

Since the lower component of all states [Eq. (27)] is similar, but their upper components have different angular behavior, it follows from Eq. (18) that these states may interact via exchange only with themselves. Calculation of the exchange energy essentially repeats that performed in Eqs. (B6) and (B7). In the final result (B7), one simply need to replace $\Psi_{S_{1}}$ by $\phi_{0 m} / \sqrt{2}$,

$$
\varepsilon_{S}^{(m)}=\mp \frac{1}{2} \int \frac{e^{2}}{r}\left|\phi_{0 m}\right|^{2} d^{2} r= \pm \frac{(2 m) !}{(m !)^{2} 2^{2 m}} \sqrt{\frac{\pi}{2}} \frac{e^{2}}{2 l} .
$$

This formula includes Eq. (B7) as the $m=0$ case.

The exchange energy for the electrons outside the unpolarized droplet is given by the modified Eq. (B1):

$$
\begin{aligned}
\varepsilon_{M}= & \mp \sum_{m=N}^{\infty} \int \frac{e^{2}}{2\left|\mathbf{r}-\mathbf{r}^{\prime}\right|} \phi_{0, M}^{*}(\mathbf{r}) \phi_{0, m}(\mathbf{r}) \\
& \times \phi_{0, m}^{*}\left(\mathbf{r}^{\prime}\right) \phi_{0, M}\left(\mathbf{r}^{\prime}\right) d \mathbf{r} d \mathbf{r}^{\prime},
\end{aligned}
$$

where the summation over $m$ excludes the electrons from the $n=0$ Landau level from inside the large droplet $N \gg 1$. This exclusion of states with $m<N$ leads to the projection operator

$$
\begin{aligned}
\rho_{N}\left(r, r^{\prime}\right) & =\sum_{m=N}^{\infty} \phi_{0, m}(r) \phi_{0, m}^{*}\left(r^{\prime}\right) \\
& =\sum_{m=N}^{\infty} \frac{\left(z^{*} z^{\prime} / 2\right)^{m}}{2 \pi m !} e^{-|z|^{2} / 4-\left|z^{\prime}\right|^{2} / 4} .
\end{aligned}
$$

Since the function $\left|\phi_{0, m}(r)\right|$ has a very pronounced maximum at $r \approx \sqrt{2 m}$, the modified projection operator (D3) coincides with Eq. (B2) in case of both arguments larger than the droplet size $r, r^{\prime}>\sqrt{2 N}$, and vanishes fast if one argument falls inside the droplet. The simple calculation helps to quantify this observation. Consider a complex variable $v$ with $\operatorname{Re} v>0$. For $N \gg 1$, one finds

$$
e^{-v} \sum_{m=N}^{\infty} \frac{v^{m}}{m !} \approx \sum_{m=N}^{\infty} \frac{e^{-(v-m)^{2} / 2 m}}{\sqrt{2 m \pi}} \approx \frac{1}{2} \operatorname{erfc}\left(\frac{N-v}{2 \sqrt{N}}\right),
$$

where a complementary error function $\operatorname{erfc}(z)=$ $(2 / \sqrt{\pi}) \int_{z}^{\infty} e^{-t^{2}} d t$. Combining this with Eq. (B2) gives

$$
\begin{aligned}
\rho_{N}\left(r, r^{\prime}\right)= & \frac{1}{2 \pi} \exp \left\{-\frac{\left|z-z^{\prime}\right|^{2}}{4}+i \operatorname{Im} \frac{z^{*} z^{\prime}}{2}\right\} \\
& \times \frac{1}{2} \operatorname{erfc}\left(\frac{2 N-z^{*} z^{\prime}}{4 \sqrt{N}}\right) .
\end{aligned}
$$

The first exponential factor here shows that the exchange interaction is effective only at distances of the order of Larmor radius $\left|\mathbf{r}-\mathbf{r}^{\prime}\right| \sim 1$. The second factor, the error function, guaranties vanishing of the interaction inside the droplet, at $|\mathbf{r}|<\sqrt{2 N}$. 
After calculating the sum over $m$ in Eq. (D2) one still needs to perform the integration over two coordinates $\mathbf{r}, \mathbf{r}^{\prime}$. We do not see an easy way to perform these integrations in a compact form. However, the resulting behavior of the energy $\varepsilon_{M}$ is clear. For large angular momentum, the electron stays at the circle larger than the droplet radius and has the same energy the electrons at the $n=0$ Landau level have in clean graphene without impurities [Eq. (20)]. Exactly at the border of the droplet the exchange-dominated Zeeman splitting drops by half:

$$
\varepsilon_{M \gg N}=\mp \frac{e^{2}}{2 l} \sqrt{\frac{\pi}{2}}, \quad \varepsilon_{M=N}=\frac{1}{2} \varepsilon_{M \gg N} .
$$

For $M$ slightly bigger than $N$, the Zeeman splitting increases continuously between the two values, reaching the asymptotic value at $M-N \gg \sqrt{N}$.
[1] K. S. Novoselov, A. K. Geim, S. V. Morozov, D. Jiang, M. I. Katsnelson, I. V. Grigorieva, S. V. Dubonos, and A. A. Firsov, Nature (London) 438, 197 (2005).

[2] Y.Zhang, Y.-W. Tan, H. L. Stormer, and P. Kim, Nature (London) 438, 201 (2005).

[3] A. H. Castro Neto, F. Guinea, N. M. R. Peres, K. S. Novoselov, and A. K. Geim, Rev. Mod. Phys. 81, 109 (2009).

[4] V. M. Pereira, F. Guinea, J. M. B. Lopes dos Santos, N. M. R. Peres, and A. H. Castro Neto, Phys. Rev. Lett. 96, 036801 (2006).

[5] A. V. Shytov, D. A. Abanin, and L. S. Levitov, Phys. Rev. Lett. 103, 016806 (2009).

[6] D. A. Abanin, A. V. Shytov, and L. S. Levitov, Phys. Rev. Lett. 105, 086802 (2010).

[7] V. V. Cheianov, V. I. Falko, O. Syljuasen, and B. L. Altshuler, Solid State Commun. 149, 1499 (2009).

[8] V. V. Cheianov, O. Syljuasen, B. L. Altshuler, and V. Falko, Phys. Rev. B 80, 233409 (2009).

[9] V. V. Cheianov, O. Syljuasen, B. L. Altshuler, and V. I. Falko, Europhys. Lett. 89, 56003 (2010).

[10] S. Kopylov, V. Cheianov, B. L. Altshuler, and V. I. Falko, Phys. Rev. B 83, 201401(R) (2011).

[11] D. M. Basko, Phys. Rev. B 78, 115432 (2008).

[12] T. O. Wehling, M. I. Katsnelson, and A. I. Lichtenstein, Chem. Phys. Lett. 476, 125 (2009).

[13] T. O. Wehling, M. I. Katsnelson, and A. I. Lichtenstein, Phys. Rev. B 80, 085428 (2009).

[14] T. O. Wehling, S. Yuan, A. I. Lichtenstein, A. K. Geim, and M. I. Katsnelson, Phys. Rev. Lett. 105, 056802 (2010).

[15] M. Titov, P. M. Ostrovsky, I. V. Gornyi, A. Schuessler, and A. D. Mirlin, Phys. Rev. Lett. 104, 076802 (2010).

[16] P. M. Ostrovsky, M. Titov, S. Bera, I. V. Gornyi, and A. D. Mirlin, Phys. Rev. Lett. 105, 266803 (2010).

[17] J. Schelter, P. M. Ostrovsky, I. V. Gornyi, B. Trauzettel, and M. Titov, Phys. Rev. Lett. 106, 166806 (2011).

[18] V. V. Mkhitaryan and E. G. Mishchenko, Phys. Rev. B 86, 115442 (2012).

[19] S. Gattenlöhner, W.-R. Hannes, P. M. Ostrovsky, I. V. Gornyi, A. D. Mirlin, and M. Titov, Phys. Rev. Lett. 112, 026802 (2014).
[20] S. Ryu, M. Y. Han, J. Maultzsch, T. F. Heinz, P. Kim, M. L. Steigerwald, and L. E. Brus, Nano Lett. 8, 4597 (2008).

[21] D. C. Elias, R. R. Nair, T. M. G. Mohiuddin, S. V. Morozov, P. Blake, M. P. Halsall, A. C. Ferrari, D. W. Boukhvalov, M. I. Katsnelson, A. K. Geim, and K. S. Novoselov, Science 323, 610 (2009).

[22] J. S. Burgess, B. R. Matis, J. T. Robinson, F. A. Bulat, F. K. Perkins, B. H. Houston, and J. W. Baldwin, Carbon 49, 4420 (2011).

[23] J. T. Robinson, J. S. Burgess, C. E. Junkermeier, S. C. Badescu, T. L. Reinecke, F. K. Perkins, M. K. Zalalutdinov, J. W. Baldwin, J. C. Culbertson, P. E. Sheehan, and E. S. Snow, Nano Lett. 10, 3001 (2010).

[24] V. P. Gusynin and S. G. Sharapov, Phys. Rev. Lett. 95, 146801 (2005).

[25] O. V. Yazyev, Phys. Rev. Lett. 101, 037203 (2008).

[26] P. O. Lehtinen, A. S. Foster, A. Ayuela, A. Krasheninnikov, K. Nordlund, and R. M. Nieminen, Phys. Rev. Lett. 91, 017202 (2003).

[27] T. O. Wehling, K. S. Novoselov, S. V. Morozov, E. E. Vdovin, M. I. Katsnelson, A. K. Geim, and A. I. Lichtenstein, Nano Lett. 8, 173 (2008).

[28] C. Jang, S. Adam, J.-H. Chen, E. D. Williams, S. Das Sarma, and M. S. Fuhrer, Phys. Rev. Lett. 101, 146805 (2008).

[29] F. Chen, J. Xia, D. K. Ferry, and N. Tao, Nano Lett. 9, 2571 (2009).

[30] L. A. Ponomarenko, R. Yang, T. M. Mohiuddin, M. I. Katsnelson, K. S. Novoselov, S. V. Morozov, A. A. Zhukov, F. Schedin, E. W. Hill, and A. K. Geim, Phys. Rev. Lett. 102, 206603 (2009).

[31] S. Kim, J. Nah, I. Jo, D. Shahrjerdi, L. Colombo, Z. Yao, E. Tutuc, and S. K. Banerjee, Appl. Phys. Lett. 94, 062107 (2009).

[32] D. A. Abanin, P. A. Lee, and L. S. Levitov, Phys. Rev. Lett. 96, 176803 (2006).

[33] B. Jancovici, Phys. Rev. Lett. 46, 386 (1981).

[34] E. V. Gorbar, V. P. Gusynin, V. A. Miransky, and I. A. Shovkovy, Phys. Rev. B 66, 045108 (2002).

[35] A. Luican-Mayer, M. Kharitonov, G. Li, C.-P. Lu, I. Skachko, Alem-Mar B. Gonçalves, K. Watanabe, T. Taniguchi, and E. Y. Andrei, Phys. Rev. Lett. 112, 036804 (2014). 